\title{
Fratura de Tillaux não percebida e lesão por sindesmose em adultos: Redução e fixação artroscópica assistida*
}

\section{Missed Tillaux Fracture and Syndesmosis Injury in Adult: Arthroscopic Assisted Reduction and Fixation}

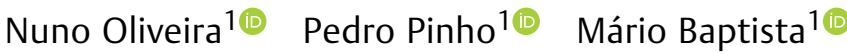 \\ Bruno S. Pereira ${ }^{1,2,3,4}$ \\ ${ }^{1}$ Serviço de Ortopedia e Traumatologia, Hospital de Braga, Sete \\ Fontes, São Victor, Braga, Portugal \\ 2 Instituto de Investigação em Ciências da Vida e Saúde (ICVS), Escola \\ de Medicina, Universidade do Minho, Braga, Portugal \\ ${ }^{3}$ Clínica do Dragão, Espregueira Mendes Sports Center, FIFA Medical \\ Center of Excellence, Estádio do Dragão, Porto, Portugal \\ 4 Universitat de Barcelona, Gran Via de les Corts Catalanes, Barcelona, Espanha \\ Rev Bras Ortop 2021;56(3):399-402.
}

Daniel Freitas ${ }^{1}$ Pedro Varanda ${ }^{1,2}$

Endereço para correspondência Nuno Oliveira, MD, Serviço de Ortopedia e Traumatologia, Hospital de Braga, Sete Fontes, SãoVictor, Braga, 4710-243, Portugal (e-mail: nunocruzoliveira1@gmail.com).

\section{Resumo \\ Palavras-chave \\ - fraturas no tornozelo \\ - lesões no tornozelo \\ - artroplastia de substituição do tornozelo \\ - procedimentos cirúrgicos minimamente invasivos}

As fraturas de Tillaux são fraturas da margem lateral da tíbia distal, geralmente relatadas em crianças entre 12 e 14 anos. Como fraturas intra-articulares, requerem redução e fixação anatômica para evitar complicações pós-traumáticas. Como o mecanismo de lesão é a rotação externa do pé na perna, essas lesões são comumente associadas a outras fraturas ou lesões ligamentares. Atualmente, a artroscopia está sendo cada vez mais utilizada para auxiliar e melhorar o tratamento cirúrgico das fraturas do tornozelo. Os autores descrevem um acompanhamento de 12 meses de um caso raro de uma fratura não percebida de Tillaux associada a lesão por sindesmose em um paciente de politrauma com 76 anos de idade, tratado com sucesso por redução e fixação interna assistida por artroscopia.

Tillaux fractures are fractures of the lateral margin of the distal tibia, usually reported in children between 12 and 14 years old. As intraarticular fractures, they require anatomic reduction and fixation to avoid posttraumatic complications. Since the injury mechanism is external rotation of the foot on the leg, these injuries are commonly associated with other fractures or ligamentous lesions. Currently, arthroscopy is being increasingly used to assist and improve surgical treatment of ankle fractures. The authors describe a 12-month follow-up of a rare case of a missed Tillaux fracture associated with syndesmosis injury in a 76-year-old polytrauma patient, successfully treated by arthroscopically-assisted reduction and internal fixation

\footnotetext{
Estudo desenvolvido no Serviço de Ortopedia e Traumatologia do Hospital de Braga, Braga, Portugal.
}

recebido

06 de Junho de 2020

aceito

06 de Julho de 2020

Publicado on-line

Dezembro 21, 2020
DOI https://doi.org/

10.1055/s-0040-1716759. ISSN 0102-3616. (c) 2020. Sociedade Brasileira de Ortopedia e Traumatologia. All rights reserved.

This is an open access article published by Thieme under the terms of the Creative Commons Attribution-NonDerivative-NonCommercial-License, permitting copying and reproduction so long as the original work is given appropriate credit. Contents may not be used for commercial purposes, or adapted, remixed, transformed or built upon. (https://creativecommons.org/ licenses/by-nc-nd/4.0/)

Thieme Revinter Publicações Ltda., Rua do Matoso 170, Rio de Janeiro, RJ, CEP 20270-135, Brazil 


\section{Introdução}

As fraturas de Tillaux são fraturas intra-articulares da tíbia distal causadas por avulsão da superfície da tíbia distal anterolateral por tração exercida no ligamento tibiofibular anterior. São lesões incomuns observadas principalmente em adolescentes com fise distal da tíbia parcialmente fechada, geralmente entre 12 e 14 anos, correspondendo a uma fratura de Salter Harris tipo III. A lesão é comumente chamada de fratura juvenil de Tillaux. ${ }^{1}$ Esse tipo de lesão é raramente observado em adultos com maturidade esquelética porque o ligamento tibiofibular anterior geralmente se rompe primeiro. ${ }^{2-5}$

As fraturas intra-articulares requerem redução e estabilização anatômicas para evitar complicações como instabilidade do tornozelo, dor no tornozelo e osteoartrite pós-traumática. ${ }^{4,6}$

A artroscopia tem várias vantagens em relação às abordagens abertas, permitindo um tratamento menos agressivo das lesões associadas, melhora da redução intra-articular e com menos complicações. ${ }^{7}$

Os autores relatam tratamento assistido por artroscopia de um caso raro de fratura de Tillaux em adulto, associado a lesão por sindesmose que foi inicialmente não percebida em um paciente com politrauma.

\section{Relato de caso}

Um homem de 76 anos foi internado no departamento de emergência após um acidente de automóvel. Ele sofreu traumatismo craniano e torácico grave, com várias fraturas de costela e do esterno, fratura não deslocada da escápula, hemopneumotórax e contusão pulmonar. Lesões na coluna e pélvica foram excluídas e o paciente foi internado em unidade de terapia intensiva (UTI). Três semanas após o trauma inicial, o paciente queixou-se de dor no tornozelo direito quando tentou levantar-se e não suportava peso. Um exame físico focalizado do tornozelo e pé direito revelou inchaço mínimo na face lateral do tornozelo, sensibilidade moderada à palpação nos maléolos laterais e restrição leve da amplitude de movimento do tornozelo. A avaliação radiológica inicial com radiografias do tornozelo mostrou uma mortise lateral alargada do tornozelo e fragmentos ósseos intraarticulares (-Figura 1). A tomografia computadorizada (TC) mostrou uma fratura de Tillaux deslocada associada ao deslocamento anterior da fíbula, sugerindo lesão por sindesmose (-Figura 2).

Sob anestesia geral, em posição supina e com torniquete aplicado à coxa proximal direita, o paciente foi submetido a artroscopia anterior do tornozelo através dos portais anteromedial e anterolateral padrão. Fibrose extensa estava presente. Após desbridamento e remoção do corpo frouxo, fraturas e lesões sindesmóticas foram facilmente visualizadas. A redução da fratura foi obtida sob visualização direta e fixada com um parafuso interfragmentar canulado de 3,0 mm, colocado através do portal anterolateral. A lesão sindesmótica foi reduzida e fixada com dois parafusos corticais trans-sindemóticos percutâneos de $4,5 \mathrm{~mm}$. A exploração artroscópica após a fixação confirmou redução

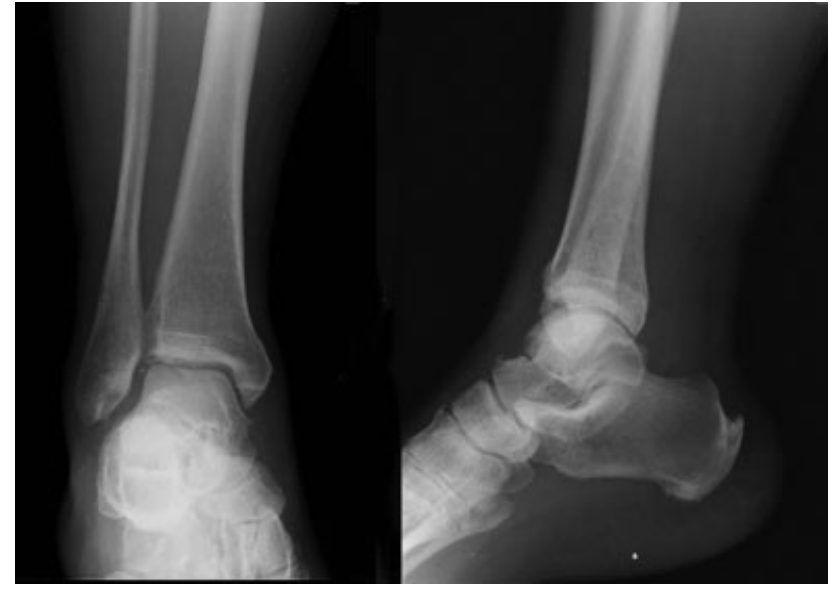

Fig. 1 Radiografia inicial padrão do tornozelo direito.

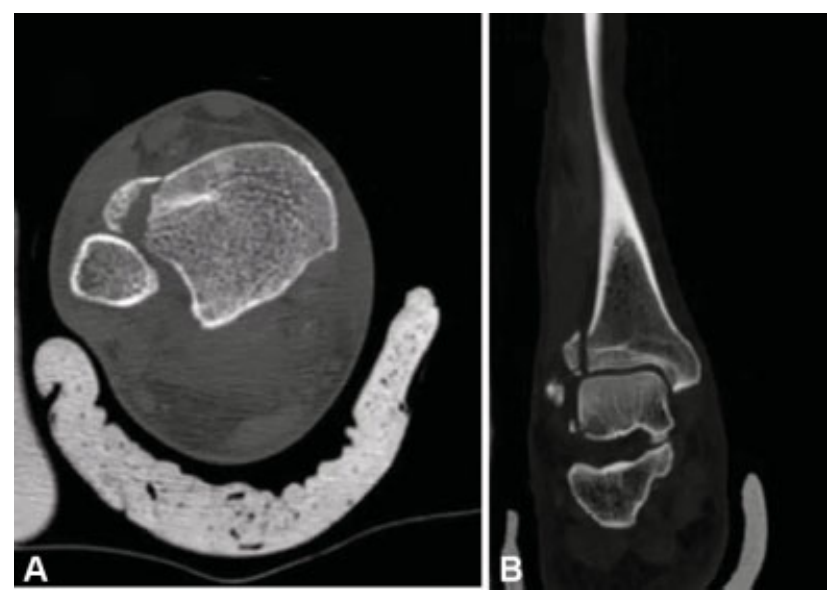

Fig. 2 Tomografia computadorizada de tornozelo direito. (A) Vista axial; (B) Vista sagital.

satisfatória, o que também foi confirmado com fluoroscopia (-Figs. 3 e 4, vídeo 1). Nenhuma imobilização foi utilizada após a cirurgia, permitindo mobilização passiva e ativa precoce.

\section{Vídeo 1}

Destaques do procedimento artroscópico.

Os parafusos trans-sindemóticos foram removidos após 8 semanas de acompanhamento e o paciente começou a suportar o peso.

Aos 6 meses, o paciente era assintomático com amplitude de movimento simétrica do tornozelo e sem limitações durante as atividades diárias (-Fig.5). 0 acompanhamento continuou por até 12 meses e nenhuma complicação foi relatada. 


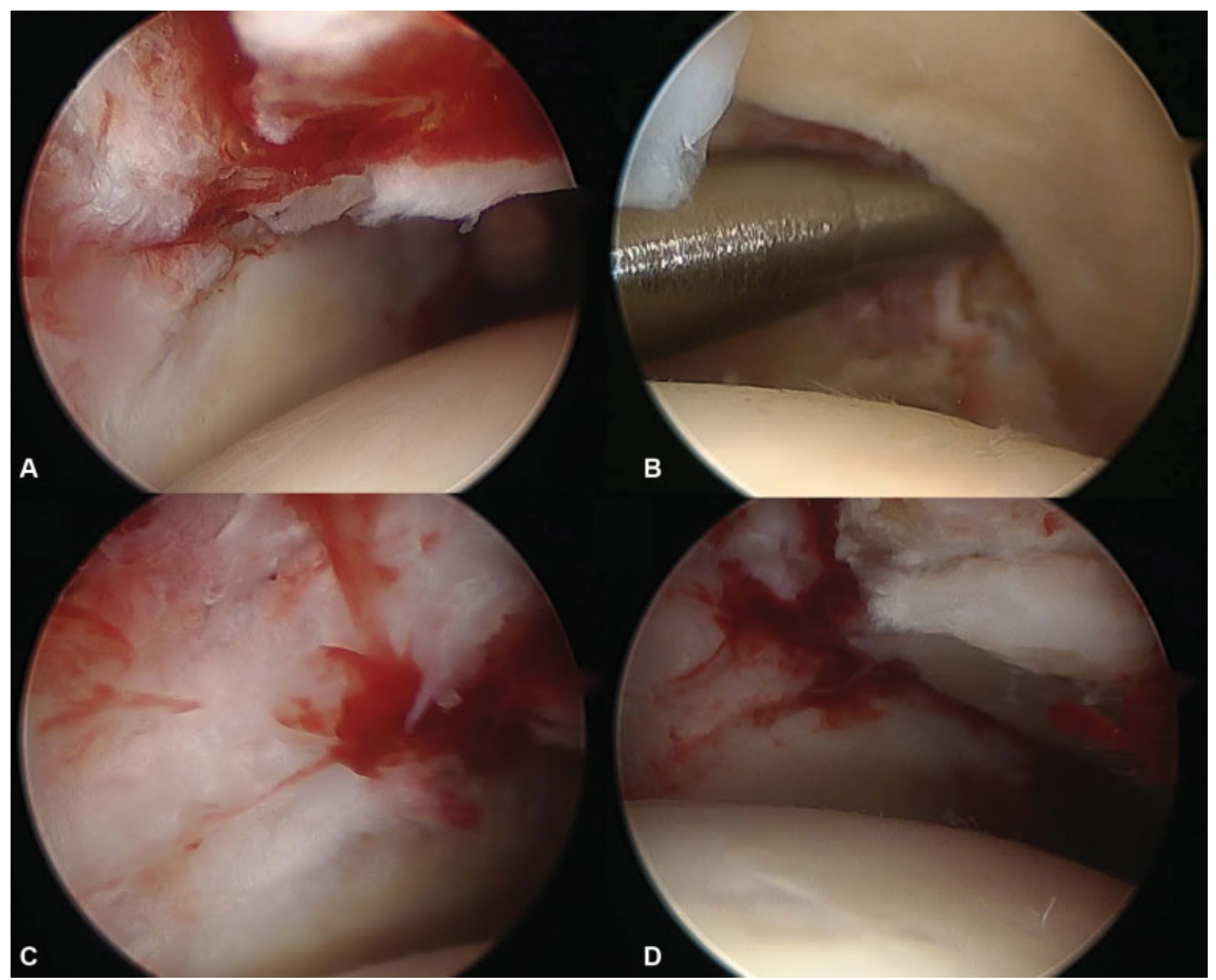

Fig. 3 Vista artroscópica. (A) Deslocamento da fratura e incongruência da articulação do tornozelo; (B) Alargamento da sindesmose; (C) Fratura após redução e fixação; (D) Sindesmose após redução e fixação.

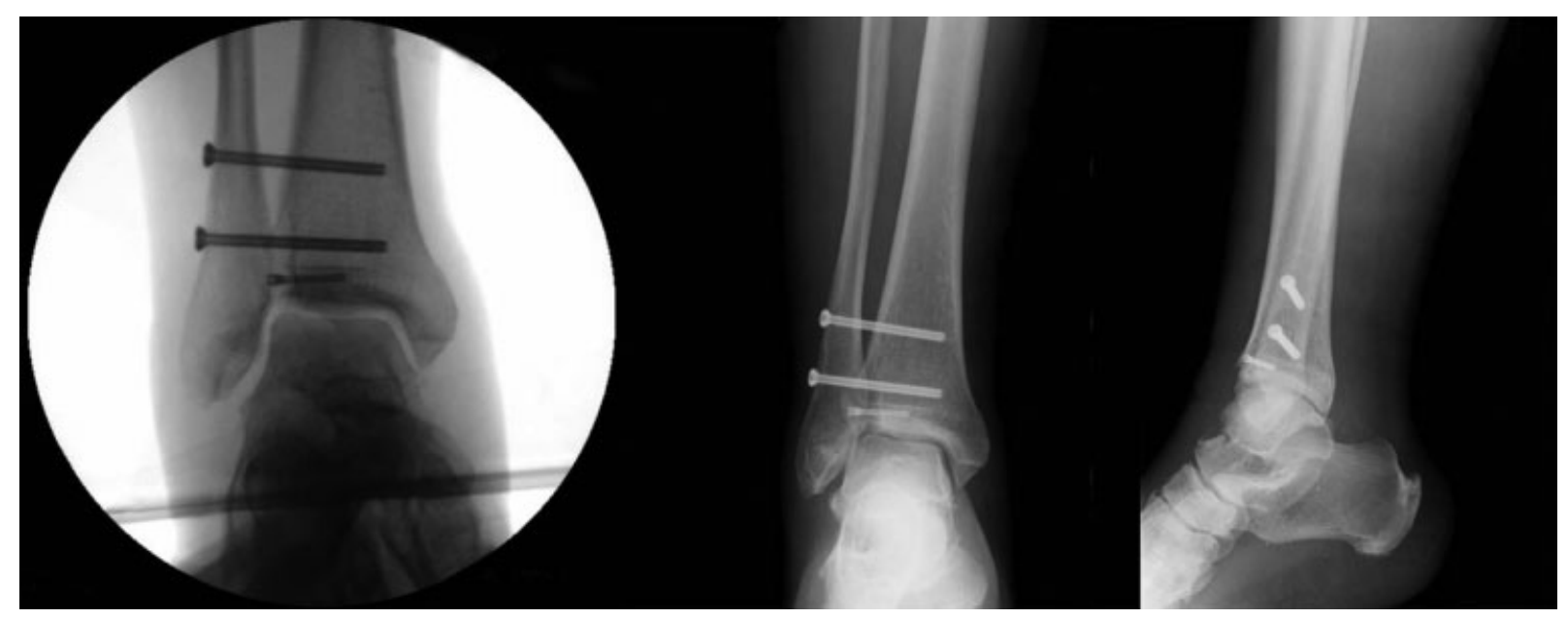

Fig. 4 Controle fluoroscópico intraoperatório - esquerdo; Radiografia pós-operatória - direita.

\section{Discussão}

O mecanismo de lesão da fratura de Tillaux é a força rotacional externa do pé na perna, que faz do ligamento tibiofibular ântero-inferior a força deformadora. ${ }^{1}$ Embora isso às vezes ocorra em adolescentes com fise parcialmente fechada, é muito raro em adultos esqueleticamente maduros. Quando há menos de $2 \mathrm{~mm}$ de deslocamento e a lesão por sindesmose é excluída, essas fraturas podem ser gerenciadas com tratamento conservador por imobilização em um molde sem suporte de peso ou tornozeleiras de suporte. As fraturas deslocadas acima de $2 \mathrm{~mm}$ devem ser tratadas cirurgicamente. ${ }^{4,6}$ 


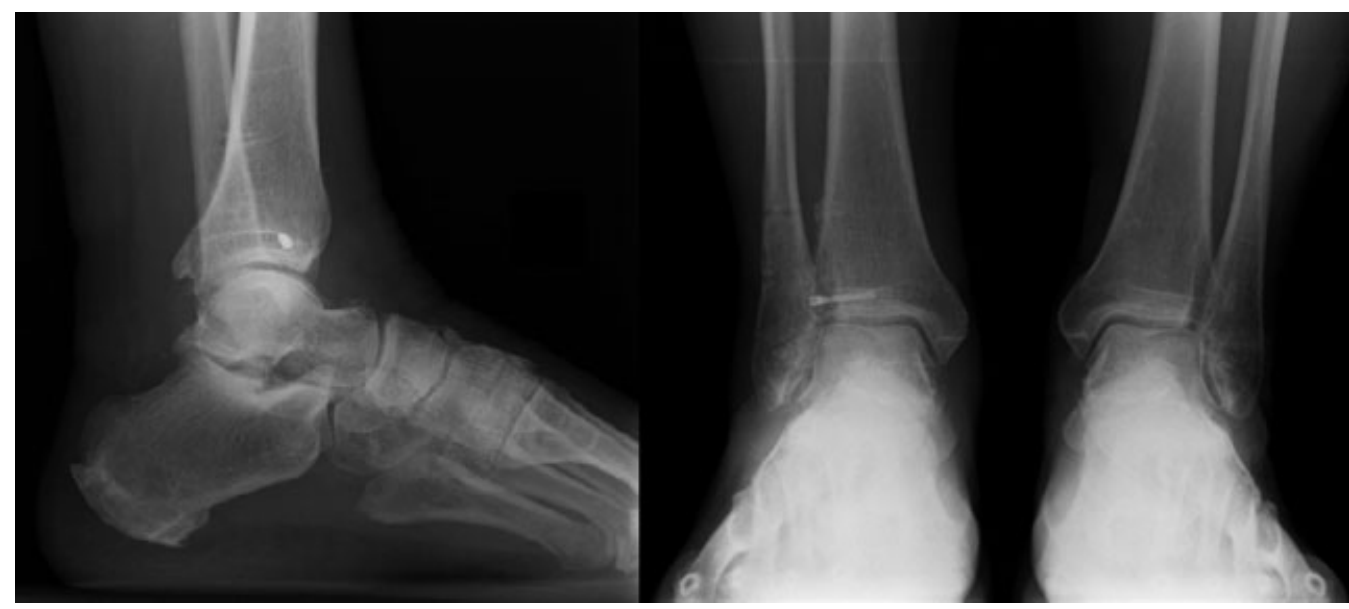

Fig. 5 Radiografia com suporte de peso aos 6 meses de acompanhamento.

Os fragmentos de fratura podem ser pequenos e podem não ser percebidos nas radiografias tradicionais do tornozelo, podendo ser necessárias incidências oblíquas. A tomografia computadorizada é mais precisa que as radiografias simples e permite uma melhor avaliação do deslocamento da fratura, padrão e congruência articular. ${ }^{6}$

A artroscopia tem sido cada vez mais usada para auxiliar o tratamento cirúrgico de fraturas do tornozelo e para gerenciar complicações pós-traumáticas. ${ }^{7}$ É uma ferramenta valiosa porque permite a visualização direta da superfície articular necessária para a redução anatômica, requer menor incisão e dissecção da pele, menos interrupções no suprimento sanguíneo ósseo e menos riscos de complicações da ferida, infecção, atraso na união e não união. Também permite o diagnóstico e o tratamento potencial de lesões associadas, como por exemplo lesões condrais. ${ }^{7-9}$ No nosso caso, além das vantagens mencionadas anteriormente, a artroscopia permitiu um desbridamento menos agressivo e mais suave do calo e do tecido fibrótico já formados três semanas após a lesão. Foi relatado que a redução aberta e a fixação interna apresentam bons a excelentes resultados em até $80 \%$ dos casos. ${ }^{10}$ Feng et al. ${ }^{6}$ relataram bons e excelentes resultados em $100 \%$ dos casos em uma série de 19 pacientes com fraturas de Tillaux tratados com suporte de artroscopia aos seis meses de acompanhamento.

Quando uma fratura da margem lateral da tíbia distal está presente, geralmente significa que os ligamentos tibiofibulares anteriores estão intactos. Entretanto, também pode ocorrer lesão por sindesmose tibiofibular. ${ }^{1,4}$ A lesão por sindesmose deve sempre ser avaliada nas fraturas do tornozelo, seja com radiografias de estresse pré-operatórias ou visualização direta intra-operatória da diástase com manobras de estresse. Nesse caso, a lesão por sindesmose foi evidente tanto nos estudos radiográficos quanto na TC préoperatória e posteriormente confirmada com visualização artroscópica direta. Exigiu um desbridamento extenso antes da redução devido a alterações fibróticas que já estavam presentes. A redução anatômica foi alcançada com o mínimo de agressão às estruturas de tecidos moles, o que proporcionou excelentes resultados e rápida reabilitação.

É importante que essas lesões sejam diagnosticadas na sala de emergência para permitir um tratamento precoce e evitar possíveis complicações. Pacientes com politraumatismo podem ter mais lesões com risco de vida que podem prejudicar a avaliação clínica de lesões periféricas, mas um exame abrangente das extremidades deve ser realizado o mais rápido possível, especialmente em lesões traumáticas de alta energia.

\section{Suporte Financeiro}

Não houve suporte financeiro de fontes públicas, comerciais, ou sem fins lucrativos.

\section{Conflito de interesses}

Os autores declaram não haver conflito de interesses.

\section{Referências}

1 Protas JM, Kornblatt BA. Fractures of the lateral margin of the distal tibia. The Tillaux fracture. Radiology 1981;138(01): 55-57

2 Miller MD. Arthroscopically assisted reduction and fixation of an adult Tillaux fracture of the ankle. Arthroscopy 1997;13(01): $117-119$

3 Kumar N, Prasad M. Tillaux fracture of the ankle in an adult: a rare injury. J Foot Ankle Surg 2014;53(06):757-758

4 Oak NR, Sabb BJ, Kadakia AR, Irwin TA. Isolated adult Tillaux fracture: a report of two cases. J Foot Ankle Surg 2014;53(04): 489-492

5 Mishra PK, Patidar V, Singh SP. Chaput Tubercle Fracture in an Adult- A Rare Case Report. J Clin Diagn Res 2017;11(03): RD01-RD02

6 Feng SM, Sun QQ Wang AG, Li CK. "All-Inside" Arthroscopic Treatment of Tillaux-Chaput Fractures: Clinical Experience and Outcomes Analysis. J Foot Ankle Surg 2018;57(01):56-59

7 Hamilton GA, Doyle MD, Castellucci-Garza FM. ArthroscopicAssisted Open Reduction Internal Fixation. Clin Podiatr Med Surg 2018;35(02):199-221

8 Leontaritis N, Hinojosa L, Panchbhavi VK. Arthroscopically detected intra-articular lesions associated with acute ankle fractures. J Bone Joint Surg Am 2009;91(02):333-339

9 Bonasia DE, Rossi R, Saltzman CL, Amendola A. The role of arthroscopy in the management of fractures about the ankle. J Am Acad Orthop Surg 2011;19(04):226-235

10 Zhao J, Shu H, Li W, Liu Y, Shi B, Zheng G. Clinical features and surgical effectiveness of ankle fractures involving Tillaux-Chaput in adults. Zhongguo Xiu Fu Chong Jian Wai Ke Za Zhi 2015;29(03): 288-291 